\title{
Validity, reliability, and calibration of the physical activity unit 7 item screener (PAU- 7S) at population scale
}

Helmut Schröder ${ }^{1,2^{*}}$ D, Isaac Subirana ${ }^{3,4}$, Julia Wärnberg ${ }^{5,6}$, María Medrano7, Marcela González-Gross ${ }^{6,8}$, Narcis Gusi ${ }^{9}$, Susana Aznar ${ }^{10,11}$, Pedro E. Alcaraz ${ }^{12,13}$, Miguel A. González-Valeiro ${ }^{14}$, Lluis Serra-Majem ${ }^{6,15,16}$, Nicolás Terrados ${ }^{17}$, Josep A. Tur ${ }^{6,18}$, Marta Segú ${ }^{19}$, Clara Homs ${ }^{20,21}$, Alicia Garcia-Álvarez ${ }^{22}$, Juan C. Benavente-Marín ${ }^{5}$, F. Javier Barón-López ${ }^{5,6}$, Idoia Labayen ${ }^{7}$, Augusto G. Zapico ${ }^{8,23}$, Jesús Sánchez-Gómez ${ }^{9}$ Fabio Jiménez-Zazo ${ }^{10}$, Elena Marín-Cascales ${ }^{12}$, Marta Sevilla-Sanchez ${ }^{14}$, Estefanía Herrera-Ramos ${ }^{15}$, Susana Pulgar ${ }^{17}$, María del Mar Bibiloni ${ }^{6,18}$, Clara Sistac-Sorigué ${ }^{19}$ and Santiago F. Gómez ${ }^{20,24^{*}}$

\section{Abstract}

Background: Validation of self-reported tools, such as physical activity (PA) questionnaires, is crucial. The aim of this study was to determine test-retest reliability, internal consistency, and the concurrent, construct, and predictive validity of the short semi-quantitative Physical Activity Unit 7 item Screener (PAU-7S), using accelerometry as the reference measurement. The effect of linear calibration on PAU-7S validity was tested.

Methods: A randomized sample of 321 healthy children aged 8-16 years (149 boys, 172 girls) from the nationwide representative PASOS study completed the PAU-7S before and after wearing an accelerometer for at least 7 consecutive days. Weight, height, and waist circumference were measured. Cronbach alpha was calculated for internal consistency. Test-retest reliability was determined by intra-class correlation (ICC). Concurrent validity was assessed by ICC and Spearman correlation coefficient between moderate to vigorous PA (MVPA) derived by the PAU-7S and by accelerometer. Concordance between both methods was analyzed by absolute agreement, weighted kappa, and Bland-Altman statistics. Multiple linear regression models were fitted for construct validity and predictive validity was determined by leave-one-out cross-validation.

\footnotetext{
*Correspondence: hschroeder@imim.es; sgomez@gasolfoundation.org

'Ciber Epidemiology and Public Health (CIBERESP), Instituto de Salud Carlos

III, Madrid, Spain

${ }^{20} \mathrm{Gasol}$ Foundation, 08830 Sant Boi de Llobregat, Spain

Full list of author information is available at the end of the article
}

(c) The Author(s). 2021 Open Access This article is licensed under a Creative Commons Attribution 4.0 International License, which permits use, sharing, adaptation, distribution and reproduction in any medium or format, as long as you give appropriate credit to the original author(s) and the source, provide a link to the Creative Commons licence, and indicate if changes were made. The images or other third party material in this article are included in the article's Creative Commons licence, unless indicated otherwise in a credit line to the material. If material is not included in the article's Creative Commons licence and your intended use is not permitted by statutory regulation or exceeds the permitted use, you will need to obtain permission directly from the copyright holder. To view a copy of this licence, visit http://creativecommons.org/licenses/by/4.0/. The Creative Commons Public Domain Dedication waiver (http://creativecommons.org/publicdomain/zero/1.0/) applies to the data made available in this article, unless otherwise stated in a credit line to the data. 
Results: The PAU-7S overestimated MVPA by 18\%, compared to accelerometers (106.5 \pm 77.0 vs $95.2 \pm 33.2 \mathrm{~min} /$ day, respectively). A Cronbach alpha of 0.76 showed an acceptable internal consistency of the PAU-7S. Test-retest reliability was good (ICC $0.71 p<0.001$ ). Spearman correlation and ICC coefficients of MVPA derived by the PAU-7S and accelerometers increased from 0.31 to 0.62 and 0.20 to 0.62 , respectively, after calibration of the PAU-7S. Between-methods concordance improved from a weighted kappa of 0.24 to 0.50 after calibration. A slight reduction in ICC, from 0.62 to 0.60 , yielded good predictive validity. Multiple linear regression models showed an inverse association of MVPA with standardized body mass index $(\beta-0.162 ; p<0.077)$ and waist to height ratio $(\beta-$ $0.010 ; p<0.014)$. All validity dimensions were somewhat stronger in boys compared to girls.

Conclusion: The PAU-7S shows a good test-retest reliability and acceptable internal consistency. All dimensions of validity increased from poor/fair to moderate/good after calibration. The PAU-7S is a valid instrument for measuring MVPA in children and adolescents.

Trial registration: Trial registration number ISRCTN34251612.

Keywords: Children and adolescents, Self-reported physical activity, Short PAQ, Validation, Accelerometry

\section{Introduction}

Physical activity (PA) is associated with favorable mental and physical health in children and adolescents [1-3]. The World Health Organization (WHO) recommends at least 60 min per day of moderate and vigorous physical activity (MVPA) for children aged 5 to 17 years [4]. This recommendation is shared by most European countries, including Spain [5].

Measurement of daily PA is paramount to identify children not meeting current recommendations and implement intervention programs aimed to promote PA that can engage this at-risk population. However, the measurement of true PA is challenging. Objective methods to measure PA, such as accelerometry, are difficult to implement in large-scale epidemiological studies and time-limited settings due to economic and logistic burdens [6, 7]. Additionally, PA measurement by accelerometers has several limitations making it difficult to compare data between studies [8]. In comparison, the administration of PA questionnaires is a cheaper and more feasible method, albeit less accurate, to meet the challenge of measuring PA in children and adolescents. These questionnaires vary in their design and structure (e.g., recorded periods of PA range from 1 day to 1 year) [7] and have been validated in specific population subgroups, which limits the transferability of results. Additionally, most questionnaires for children and adolescents include qualitative questions, ask for details about PA frequencies, and are generally too complex for use in time-limited settings such as the pediatrician's daily practice [7]. Several short quantitative PA questionnaires are available but were validated in specific populations [9-12], which limits their use in other populations. Furthermore, these questionnaires are limited for cross-nation comparison of PA. Therefore, brief PA questionnaires are needed to readily identify children not meeting the WHO PA recommendations and assist in PA counseling [13]. For this reason we developed the Physical Activity Unit 7-day Screener (PAU-7S), a brief PA questionnaire developed to measure PA in children and adolescents.

PA questionnaires are generally developed specifically for each study population and research aim due to the impact of ethnicity, culture, behavior, and biology on PA (7). Therefore, the validity of PA questionnaires beyond the target study population is limited. Furthermore, measurement error of self-reported data is a concern. The available calibrated questionnaire-derived PA data, although scant, show promising results [11, 14-18].

The aim of the present study was to determine the reliability and the concurrent, construct, and predictive validity of PAU-7S in a randomized, nationally representative subsample of the PASOS study of children and adolescents aged 8 to 16 years. Additionally, we evaluated the effect of linear regression calibration on each of the three dimensions of PAU-7S validity.

\section{Methods \\ Participants}

This validation study was performed within the frame of a nationwide representative study of Physical Activity, Sedentarism, lifestyles and Obesity in Spanish youth (PASOS). The methodology of the PASOS study has been described in detail elsewhere [19]. In brief, a representative sample from 22 school groups of 4508 children aged 8-16 years and their parents were invited, of whom 3817 agreed to participate ( $84.7 \%$ response rate).

Of this study population, a randomized sample of 389 $(10.2 \%)$ children and adolescents was invited to participate in the validation study and 369 (94.9\% response rate) agreed to participate. For test-retest reliability analysis, 321 participants completed PAU-7S questionnaires at baseline and after 1 week of wearing an accelerometer. After excluding 17 participants with missing or invalid 
accelerometer data, 304 participants were included in the validity analysis. Participants did not receive any compensation for their participation. The study protocol was approved by the Ethics Committee CEIm Fundació Sant Joan de Déu, Spain (Approval number: PIC-17118). Parental written informed consent was obtained.

\section{Development and administration of the PAU-7S}

The development of the PAU-7S involved three strategies: (i) review of validated PA questionnaires for children; (ii) consulting PA experts from the IMIM-Hospital del Mar Research Institute; (iii) analysis of PA data of the Thao-POIBC [20] and EnKid [21] studies to identify activities that explain the variability of PA in children and young adolescents.

The resulting 7-day PA questionnaire was designed to measure regular PA in a typical week. Most PA questionnaires commonly used with children focus on this timeframe because they show good weekly recall [22]. The PAU-7S questionnaire design considered the usual opportunities to do PA during the day. The online questionnaire included 6 main questions about the previous week: 1 . How many days did you go for a walk? 2. How many days were you engaged in active play during recess time? 3. How many days were you engaged in active play during free time after school or during the weekend? 4. How many days did you have Physical Education (PE) class at school? 5 . How many days did you play a team sport? (for example: soccer, basket, handball, hockey, and water polo). 6 . How many days did you play individual sports? (for example: track and field, eurythmics, dance-ballet, tennis, judo-karate-taekwondo, rollerskating, swimming). For each question, the answering options were presented as a table showing each day with spaces where the children would mark if they spent (i) less than $30 \mathrm{~min}$ on the activity that day; (ii) $30 \mathrm{~min}$ to one hour; (iii) one hour to one hour and a half; or (iv) more than one hour and a half. Children had to select an option before progressing through the online system. For the second and fourth questions, which ask about physical activity during school time, response options were only shown from Monday to Friday; question 4 did not include time options because a PE class lasts $45 \mathrm{~min}$ in Spain. Additionally, two qualitative items were included i) Are any of these sports aquatic activities? (Yes/ $\mathrm{No}$ ) and ii) Were you sick during the past week or did anything prevent you from doing your usual physical activities? The first item was a sub-question of item 5 (How many days did you play a team sport?) and item 6 (How many days did you play individual sports?) The qualitative questions were not used to calculate MVPA.

The PAU-7S was administered the first day the accelerometer was worn and 9 days later, when the accelerometer was taken off by trained personnel. MVPA was calculated based on the sum of all activities, with the exception of walking.

\section{Physical activity measured by accelerometry}

PA was measured by the "Actigraph GT3X+" accelerometer (ActiGraph, Pensacola FL- USA), allocating at least 7 days from April to June 2019 for each of the 22 randomized school groups. Children were asked to wear the accelerometer for at least 1 week except while bathing or swimming. The accelerometers were placed on the wrist for the non-dominant hand with a bracelet. The accelerometer data collection protocol was followed by all field workers. A common training session was carried out to ensure the homogeneity of this procedure. The Troiano et al. method [23] was used to identify the time that accelerometers were not worn: periods of $60 \mathrm{~min}$ (or more) of zero values were discarded. Data from the accelerometers were considered valid if the accelerometer was worn for at least 4 days with at least 1 weekend day and for at least $10 \mathrm{~h}$ between $8 \mathrm{a} . \mathrm{m}$. and $10 \mathrm{p} . \mathrm{m}$. each day. The sampling period was set to 5 epochs $(100 \mathrm{~Hz})$ and the outcome was expressed as minutes per day. Chandler et al. cut-off points [24] were used to translate acceleration counts into minutes per week of sedentary, light, moderate, and vigorous PA.

The Actigraph data were downloaded using the software provided by the manufacturer (version 6.0, Actigraph, Pensacola, Florida) and imported into SPSS v21 (IBM, Chicago, IL) for data processing and screening. $\mathrm{R}$ package 4.0.2 accelerator (www.datahunter.es) was used to identify wear-time between 8 a.m. and 10 p.m.

\section{Anthropometric variables}

Weight, height, and waist circumferences (WC) measurements were taken by trained personnel, with the children in light clothing, without shoes. The measurements were performed using an electronic SECA 899 scale (recorded to the nearest $100 \mathrm{~g}$ ), a portable SECA 217 stadiometer (to the nearest $1 \mathrm{~mm}$ ), and a flexible, non-stretch SECA 201 metric tape (to the nearest 1 $\mathrm{mm})$, respectively. WC was measured in the narrowest zone between the lower costal rib and iliac crest, in the supine decubitus and horizontal positions. BMI z-score was computed using age and sex-specific reference values from the WHO [25]. Waist to-height ratio (WHtR) was calculated.

\section{Data collection procedure}

Following anthropometric and initial weight, height, and WC measurements, participants completed the first PAU-7S during a group session in the computer room at school (1st PAU-7S). Upon questionnaire completion, they received an accelerometer and verbal instructions 
on its use. Nine days later, participants again completed the questionnaire (2nd PAU-7S).

\section{Statistical analysis}

Participant characteristics were described as mean, standard deviation (SD), and median (inter-quartile range), as appropriate. Distribution of continuous variables between boys and girls were compared using the Student $\mathrm{t}$ test for normally distributed variables or Mann-Whitney U test otherwise. Proportion comparisons for categorical variables were assessed using chisquare test. Non-normally distributed variables were logtransformed to achieve normality.

Internal consistency of the PAU-7S questionnaire was tested by Cronbach alpha. Test-retest reliability between PAU-7S-derived basal and 1-week MVPA data was assessed by intra-class correlation coefficients (ICC).

The relative validity of the PAU-7S was assessed by Pearson correlation coefficients comparing MVPA derived by the PAU-7S (test method) to that shown by the accelerometers (criterion standard for validity). Pearson correlation coefficients were classified as follows: $>0.8$, very good; $0.61-0.80$, good; 0.41-0.60, moderate; 0.21-0.40, fair; and $<=0.20$, weak [26]. Although the two measurements might be highly correlated, substantial differences between them could exist across the range of values; therefore, we determined absolute agreement between the two measurements by cross-classification and the kappa statistic of tercile distribution of MVPA for both measurements. Concordance between the PAU-7S measurements of MVPA was assessed by kappa values as follows: $>0.8$, almost perfect agreement; 0.61-0.80, substantial agreement; $0.41-0.60$, moderate agreement; $0.21-0.40$, fair agreement; and $<=0.20$, slight agreement (24).

We further assessed agreement between the two measurements using the original Bland-Altman method [27] and a modified version published by Ludbrook [28]. Both methods calculate the mean of differences between the two measurements and regress it against the mean obtained with each measurement. The method by Ludbrook assumes a possible bias as a function of the mean of each participant and computes the confidence limits accordingly. A mean proportional agreement of 100\% between measurements would signify complete agreement; a mean difference of 0 would show complete disagreement between the methods. In addition, we analyzed possible variations in the level of agreement between methods to assess proportional bias. For this purpose, we fitted linear regression models, with the mean instrument differences of MVPA derived by the PAU-7S and accelerometers (MVPA_PAU-7S - MVPAaccelerometers)) as the dependent variable and the mean score of both ((MVPA_PAU-7S + MVPA_accelerometer) / 2)) as the independent variable.
Energy balance is the ratio between energy intake and energy expenditure. Energy expenditure increases with PA [29, 30], which might compensate for excessive energy and its effect on weight gain. Therefore, we hypothesized that a valid construct of the PAU-7S would be inversely associated with body mass index (BMI) and WC. Multiple linear regression models adjusted for sex and age, with anthropometric variables as the outcome and MVPA derived by the PAU-7S as the exposure, were fitted to test construct validity of the PAU-7S. All models were tested for multicollinearity.

To reduce measurement error of the PAU-7S, we performed linear calibration according to Rosner et al. [31]. MVPA derived by accelerometers was regressed against MVPA obtained by the PAU-7S with sex, age, and weight as co-variables. The final calibrated model (MVPA_PAU7S_calibrated) was as follows: MVPA_PAU-7S_calibrated $=183.788$ (intercept $)+(-6.374 *$ age $)+(1.437 *$ sex $)+\left(0.080^{*}\right.$ MVPA_PAU-7S $)+(-0.436 "$ weight $)$.

The predictive capacity of the calibration equation was assessed by leave-one-out cross-validation. This iterative procedure predicts the response value of each individual from the model fitted by the rest of the sample. The classification system of interaction between the PAU-7Sderived MVPA, sex, and age was tested.

The Statistical Package for the Social Sciences statistical software package version 21.0 (SPSS Inc., Chicago, IL, USA) was used for all statistical analyses with the exception of leave-one-out cross-validation. This analysis was performed using $\mathrm{R}$ package 4.0.2. Differences were considered significant if $p<0.05$.

\section{Results}

Characteristics of the study population are reported in Table 1. Girls were slightly older, with a higher BMI, compared to boys. At baseline, boys reported higher total PA and MVPA and more minutes spent in team sports and active play outside of school, compared to girls. There was no significant interaction between MVPA derived by the PAU-7S, sex, and age. The comparison of the general characteristics between participants of the validation study $(n=323)$ and those of the remaining PASOS cohort $(n=3496)$ revealed no significant differences with the exception of age. Participants in the validation study were somewhat younger (12.3 \pm 2.2 years) than the remaining participants of the PASOS cohort (12.6 \pm 2.4$)$ (Supplementary Table 1). No significant difference between the sample with accelerometer data $(n=304)$ and the sample for reliability analysis $(n=$ 321) was found.

The PAU-7S showed good test-retest repeatability for total PA and MVPA in both boys and girls (Table 2). The repeatability of each PA activity ranged from moderate to good. 
Table 1 Characteristics of the study population ${ }^{a}$

\begin{tabular}{|c|c|c|c|c|}
\hline & All & Boys & Girls & $p^{\mathbf{b}}$ \\
\hline & $(n=321)$ & $(n=149)$ & $(n=172)$ & \\
\hline Age (years) & $12.3(2.21)$ & $12.0(2.24)$ & $12.5(2.15)$ & 0.017 \\
\hline Weight (kg) & $47.6(14.6)$ & $46.4(14.4)$ & $48.6(14.8)$ & 0.174 \\
\hline Height (cm) & $1.52(0.13)$ & $1.52(0.15)$ & $1.52(0.11)$ & 0.917 \\
\hline $\mathrm{BMI}\left(\mathrm{kg} / \mathrm{m}^{2}\right)$ & $20.2(4.03)$ & $19.7(3.46)$ & $20.6(4.42)$ & 0.031 \\
\hline BMl z score & $0.57(1.25)$ & $0.61(1.27)$ & $0.53(1.24)$ & 0.589 \\
\hline Waist circumference $(\mathrm{cm})$ & $70.0(10.6)$ & $70.2(10.5)$ & $69.8(10.7)$ & 0.700 \\
\hline $\mathrm{WH} \mathrm{tR}(\mathrm{cm} / \mathrm{cm})$ & $0.46(0.06)$ & $0.46(0.06)$ & $0.46(0.06)$ & 0.494 \\
\hline \multicolumn{5}{|l|}{ 1st PAU-7S questionnaire } \\
\hline Total physical activity (min/d) & $167(92.8)$ & $182(93.1)$ & $154(90.8)$ & 0.007 \\
\hline $\operatorname{MVPA}(\mathrm{min} / \mathrm{d})^{3}$ & $120(76.6)$ & $134(77.5)$ & $108(73.8)$ & 0.002 \\
\hline Walking (min/d) & $40.7[23.6 ; 66.4]$ & $45.0[27.9 ; 66.4]$ & $40.7[23.6 ; 66.4]$ & 0.511 \\
\hline Schoolyard active play $(\mathrm{min} / \mathrm{d})^{c}$ & $10.7[10.7 ; 32.1]$ & $10.7[10.7 ; 32.1]$ & $10.7[8.04 ; 23.6]$ & 0.067 \\
\hline Non-school active play $(\mathrm{min} / \mathrm{d})^{\mathrm{d}}$ & $38.6[17.1 ; 60.0]$ & $45.0[21.4 ; 68.6]$ & $32.1[15.0 ; 53.6]$ & 0.017 \\
\hline Physical education (min/d) & $4.29[4.29 ; 4.29]$ & $4.29[4.29 ; 4.29]$ & $4.29[4.29 ; 4.29]$ & 0.133 \\
\hline Team sport $(\mathrm{min} / \mathrm{d})$ & $27.9[4.29 ; 51.4]$ & $34.3[12.9 ; 55.7]$ & $15.0[0.00 ; 45.0]$ & $<0.001$ \\
\hline Individual sport (min/d) & $15.0[0.00 ; 40.7]$ & $12.9[0.00 ; 45.0]$ & $16.1[2.14 ; 36.4]$ & 0.707 \\
\hline \multicolumn{5}{|l|}{ 2nd PAU-7S questionnaire } \\
\hline Total physical activity (min/d) & $151(95.1)$ & $159(96.4)$ & $144(93.6)$ & 0.579 \\
\hline MVPA (min/d) & $107(78.2)$ & $114(79.2)$ & $101(77.1)$ & 0.135 \\
\hline Walking (min/d) & $40.7[23.6 ; 62.1]$ & $45.0[23.6 ; 62.1]$ & $36.4[23.6 ; 57.9]$ & 0.579 \\
\hline Schoolyard active play $(\mathrm{min} / \mathrm{d})^{c}$ & $10.7[6.43 ; 32.1]$ & $10.7[10.7 ; 32.1]$ & $10.7[0.00 ; 28.9]$ & 0.049 \\
\hline Non-school active play $(\mathrm{min} / \mathrm{d})^{\mathrm{d}}$ & $32.1[15.0 ; 57.9]$ & $32.1[15.0 ; 60.0]$ & $32.1[12.9 ; 54.1]$ & 0.333 \\
\hline Physical education (min/d) & $4.29[2.14 ; 4.29]$ & $4.29[4.29 ; 4.29]$ & $4.29[2.14 ; 4.29]$ & 0.009 \\
\hline Team sport $(\mathrm{min} / \mathrm{d})$ & $15.0[0.00 ; 42.9]$ & $25.7[0.00 ; 45.0]$ & $12.9[0.00 ; 36.4]$ & 0.039 \\
\hline Individual sport (min/d) & $12.9[0.00 ; 36.4]$ & $10.7[0.00 ; 32.1]$ & $15.0[0.00 ; 39.1]$ & 0.180 \\
\hline
\end{tabular}

BMI: Body mass index; MVPA: Moderate to vigorous physical activity; WHtR: Waist to height ratio

a Variables are expressed as mean (SD) or median [interquartile range] for normal and non-normal continuous variables, respectively

${ }^{\mathrm{b}}$ Gender differences were analyzed by Student $\mathrm{t}$ test for independent variables

'Physical activity during school recess

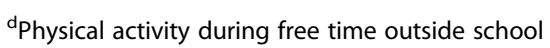

Table 2 Test-retest repeatability ${ }^{\mathrm{a}}$ of physical activity (PA) recorded by PAU-7S at baseline and after 1 week $^{\mathrm{b}}$

\begin{tabular}{|c|c|c|c|}
\hline & All & Boys & Girls \\
\hline & $(n=321)$ & $(n=149)$ & $(n=172)$ \\
\hline Total PA (min/d) & 0.73 & 0.74 & 0.72 \\
\hline Moderate to vigorous PA $(\mathrm{min} / \text { day })^{c}$ & 0.71 & 0.71 & 0.70 \\
\hline Walking $(\mathrm{min} / \mathrm{d})^{\mathrm{d}}$ & 0.69 & 0.70 & 0.68 \\
\hline Schoolyard active play $(\mathrm{min} / \mathrm{d})^{\mathrm{d}, \mathrm{e}}$ & 0.47 & 0.48 & 0.45 \\
\hline Physical education $(\mathrm{min} / \mathrm{d})^{d}$ & 0.77 & 0.74 & 0.78 \\
\hline Non-school active play $(\mathrm{min} / \mathrm{d})^{\mathrm{d}, \mathrm{f}}$ & 0.61 & 0.60 & 0.62 \\
\hline Team sport $(\mathrm{min} / \mathrm{d})^{\mathrm{d}}$ & 0.52 & 0.55 & 0.47 \\
\hline Individual sport $(\mathrm{min} / \mathrm{d})^{\mathrm{d}}$ & 0.68 & 0.61 & 0.73 \\
\hline
\end{tabular}

${ }^{a}$ Intra-class correlation coefficient

b $p<0.001$ for all correlations

c Walking was excluded

${ }^{d}$ Log-transformed before analysis

e Physical activity during school recess

${ }^{\mathrm{f}}$ Physical activity during free time outside school
The Cronbach alpha of 0.76 indicated acceptable internal consistency of the PAU-7S, with a slightly better result in girls than in boys (Table 3). The noncalibrated PAU-7S significantly overestimated MVPA (by 18\%) compared to the criterion standard for validity; furthermore, this discrepancy significantly increased ( $\beta$ coefficient $0.428(0.379 ; 0.478)$ with higher levels of MVPA (Table 3 and Fig. 1). Overestimation of MVPA was somewhat greater in boys than in girls (Table 3) and in adolescents compared to children (Supplementary Table 2). Table 3 also shows Pearson correlation coefficients between methods, indicating the capacity of the PAU-7S to rank levels of MVPA in children. Pearson coefficients between MVPA derived by the PAU-7S and by accelerometers revealed a fair concurrent validity of the PAU-7S overall as well as separately for boys and girls and for children and adolescents (Supplementary Table 2. 
Table 3 Correlation coefficients and between-method agreement of moderate to vigorous physical activity (MVPA) measurements derived by the Physical Activity Unit 7 item screener, noncalibrated and calibrated, and the reference method (accelerometer)

\begin{tabular}{|c|c|c|c|}
\hline & All $(n=304)$ & Boys $(n=141)$ & Girls $(n=163)$ \\
\hline \multicolumn{4}{|l|}{ Accelerometer } \\
\hline MVPA, $\min / d(S D)$ & $95.2(33.2)$ & $97.2(33.8)$ & $93.4(32.7)$ \\
\hline \multicolumn{4}{|l|}{$\underline{\text { PAU-7S, noncalibrated }}$} \\
\hline MVPA, min/d (SD) & $106.5(77.0)$ & $111.0(77.2)$ & $102.7(76.9)$ \\
\hline Between-method difference, min/d $(95 \% \text { Cl) })^{a}$ & $11.4(2.9 ; 19.8)$ & $13.8(1.5 ; 26.0)$ & $9.3(-2.5 ; 21.1)$ \\
\hline Proportional agreement, \%; $(95 \% \text { Cl) })^{\mathrm{b}}$ & $118(107 ; 128)$ & $120(106 ; 134)$ & $116(101 ; 131)$ \\
\hline Cronbach alpha & 0.76 & 0.73 & 0.78 \\
\hline Regression coefficient $^{c}$ & $0.428(0.379 ; 0.478)$ & $0.444(0.367 ; 0.520)$ & $0.414(0.349 ; 0.479)$ \\
\hline Spearman correlation coefficient & 0.31 & 0.33 & 0.29 \\
\hline Intra-class correlation coefficient & 0.20 & 0.24 & 0.16 \\
\hline Absolute agreement, $\%^{\mathrm{d}}$ & 46.7 & 48.2 & 45.4 \\
\hline Gross misclassification, ${ }^{\mathrm{e}}$ & 14.1 & 14.9 & 13.5 \\
\hline Kappa $^{f}$ & 0.24 & 0.24 & 0.24 \\
\hline$\underline{\text { PAU-7S, calibrated }}$ & $95.2(33.2)$ & $97.2(33.8)$ & $93.4(32.7)$ \\
\hline Between-method difference, $\min / d(95 \% \mathrm{Cl})^{\mathrm{a}}$ & $0(-3.3 ; 3.3)$ & $1.3(-3.4 ; 5.6)$ & $-1.09(-5.6 ; 3.5)$ \\
\hline Proportional agreement, \%; $(95 \% \mathrm{Cl})^{\mathrm{b}}$ & $105(101 ; 109)$ & $106(100 ; 112)$ & $104(99 ; 110)$ \\
\hline Regression coefficient ${ }^{c}$ & $1.9^{*} 10^{-6}(-0.116 ; 0.116)$ & $0.007(-0.177 ; 0.192)$ & $-0.012(-0.160 ; 0.137)$ \\
\hline Spearman correlation coefficient & 0.62 & 0.66 & 0.57 \\
\hline Intra-class correlation coefficient & 0.62 & 0.66 & 0.57 \\
\hline Absolute agreement, $\%^{\mathrm{d}}$ & 59.2 & 65.2 & 54.0 \\
\hline Gross misclassification, \% ${ }^{\mathrm{e}}$ & 3.2 & 1.8 & 3.7 \\
\hline Kappa $^{f}$ & 0.50 & 0.57 & 0.44 \\
\hline
\end{tabular}

Cl: confidence interval; MVPA: moderate to vigorous physical activity; PAU-7S: Physical Activity Unit 7-item screener; SD: standard deviation

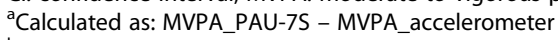

${ }^{b}$ Calculated as: [MVPA accelerometer/MVPA-PAU-7S] * 100

${ }^{c}$ Regression coefficients $(\beta)$ between mean of the MVPA and mean differences (independent variable) between MVPA obtained by the PAU-7S and

by accelerometers

${ }^{\mathrm{d}}$ Correctly classified terciles of MVPA derived by the PAU-7S and by accelerometers

epposite terciles of MVPA derived by the PAU-7S and by accelerometers

${ }^{f}$ Weighted kappa between terciles of MVPA derived by the PAU-7S and accelerometers

The absolute agreement of the PAU-7S as measured by correct cross-classification of tercile distribution of MVPA by the two methods was $46.7 \%$ for the entire population; somewhat higher values were observed for boys $(48.2 \%)$ compared to girls $(45.2 \%)$ (Table 3$)$. Additional kappa statistics, which account for agreement by chance, showed fair concordance between the two methods, with the identical kappa value in boys and girls $(\mathrm{k}=0.24)$ (Table 3).

Multiple linear regression models adjusted for sex and age revealed an inverse association of MVPA derived by PAU-7S with WHtR $(p=0.014)$ and standardized BMI (zBMI) $(p=0.077)$, as shown in Table 4. Calibration models showed a significant collinearity. Therefore, age and sex were excluded from the final model.

Concurrent validity considerably improved after linear calibration of the PAU-7S (Table 3). Pearson and ICC coefficients increased to 0.62 for both of these dimensions, with similar results in both boys and girls. The concordance between methods as measured by absolute agreement and kappa statistic improved to $59.2 \%$ and 0.50 , respectively. The regression coefficients of the MVPA association with zBMI and WHtR slightly increased after calibration of the PAU-7S (Table 4).

The mean agreement between MVPA reported by accelerometers (criterion standard for validity) is shown in Figs. 2 and 3 and the non-calibrated and calibrated PAU-7S data in Figs. 4 and 5. The non-calibrated PAU7s shows a significantly overestimation of MVPA and a significant proportional bias. The predicted difference in MVPA between the PAU7s and accelerometers increased by $0.428(p<0-05)$ and $1.9 * 10^{-6}(p>0.05)$ $\mathrm{min} / \mathrm{d}$ per each minute of the mean MVPA obtained by both methods, for the non-calibrated and calibrated 


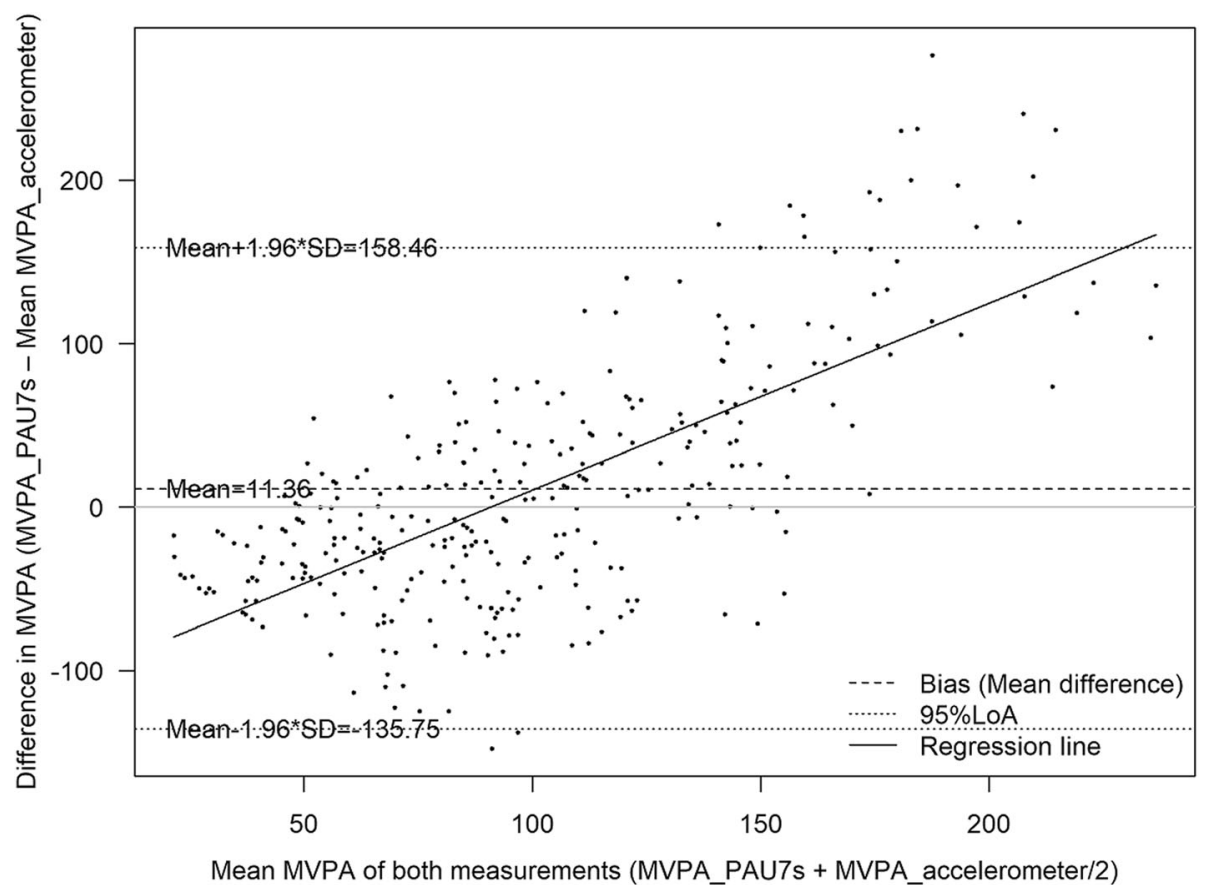

Fig. 1 Bland-Altman plot for the agreement of moderate-to-vigorous physical activity (MVPA) derived from the un-calibrated Physical Activity Unit 7 item Screener (PAU-7S) and the accelerometer $(N=301)$

PAU7s, respectively. This fact indicates that the calibration of the questionnaire eliminated the proportional bias.

Cross-validation analysis of the predictive validity of the PAU-7S showed a slight reduction of Pearson and ICC coefficients from the original validation sample to the test sample from $0.62(95 \%$ CI $0.54 ; 0.67)$ to 0.60 (95\% CI $0.55 ; 0.0 .69)$ and from 0.62 (95\% CI $0.55 ; 0.0 .69)$ to 0.60 (95\% CI $0.53 ; 0.67)$.

\section{Discussion}

The PAU-7S showed a good test-retest reliability and acceptable internal consistency. The questionnaire fairly ranked children according to levels of MVPA, with slightly better results in boys and adolescents compared

Table 4 Multiple linear regression ${ }^{\mathrm{a}}$ of the association between PAU-7S-derived moderate to vigorous physical activity and anthropometric markers of body fat

\begin{tabular}{clll}
\hline & B coefficient $^{\text {b }}$ & 95\% Cl & p \\
\hline PAU-7S, non-calibrated & & & \\
zBMI $\left(\mathrm{kg} / \mathrm{m}^{2}\right)$ & -0.162 & $-0.345 ; 0.018$ & 0.077 \\
WHtR $(\mathrm{cm} / \mathrm{cm})$ & -0.010 & $-0.019 ;-0.001$ & 0.014 \\
PAU-7S, calibrated & & & \\
zBMI $\left(\mathrm{kg} / \mathrm{m}^{2}\right)$ & -0.216 & $-0.638 ; 0.200$ & 0.316 \\
WHtR $(\mathrm{cm} / \mathrm{cm})$ & -0.025 & $-0.044 ;-0.006$ & 0.009
\end{tabular}

Cl: Confidence interval; PAU-7S: physical activity unit 7-item screener; zBMI: standardized body mass index; WHtR: waist to height ratio

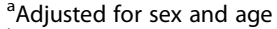

${ }^{\mathrm{b}}$ Per $100 \mathrm{~min}$ of MVPA per day to girls. Most importantly, although the noncalibrated PAU-7S overestimated MVPA, especially at higher levels of activity, linear calibration meaningfully increased the concurrent and construct validity of the questionnaire.

In general, the ability of PA questionnaires to adequately measure PA in children and adolescents is modest at best $[32,33]$. Although objective measurement of PA by accelerometry is an option, it is not always feasible due to economic and logistical burdens, and accelerometer-derived data lack information on context and type of activity (7).

The consistency of participant responses across the items of the PAU-7S was determined by Cronbach alpha. In general, all the questionnaire items are supposed to reflect the same underlying construct, and therefore should be correlated with each others [34]. The internal consistency of the PAU-7S is within an acceptable range (Cronbach alpha $=0.76$ ) [35] and comparable to that of other PA questionnaires used in youth $[36,37]$. Test-retest reliability for total PA and MVPA derived by the PAU-7S was good overall and not meaningfully different between boys and girls. The observed ICC for MVPA was lower than that of the Spanish adaptation of the Physical Activity Questionnaire for Children (PAQ-C) [37]. However, the nearly perfect testretest repeatability (ICC 0.96) of the PAQ-C is likely due to the short timeframe for the second administration of the questionnaire, within $6 \mathrm{~h}$ of baseline. A recently published work in 712 Spanish children and adolescents 


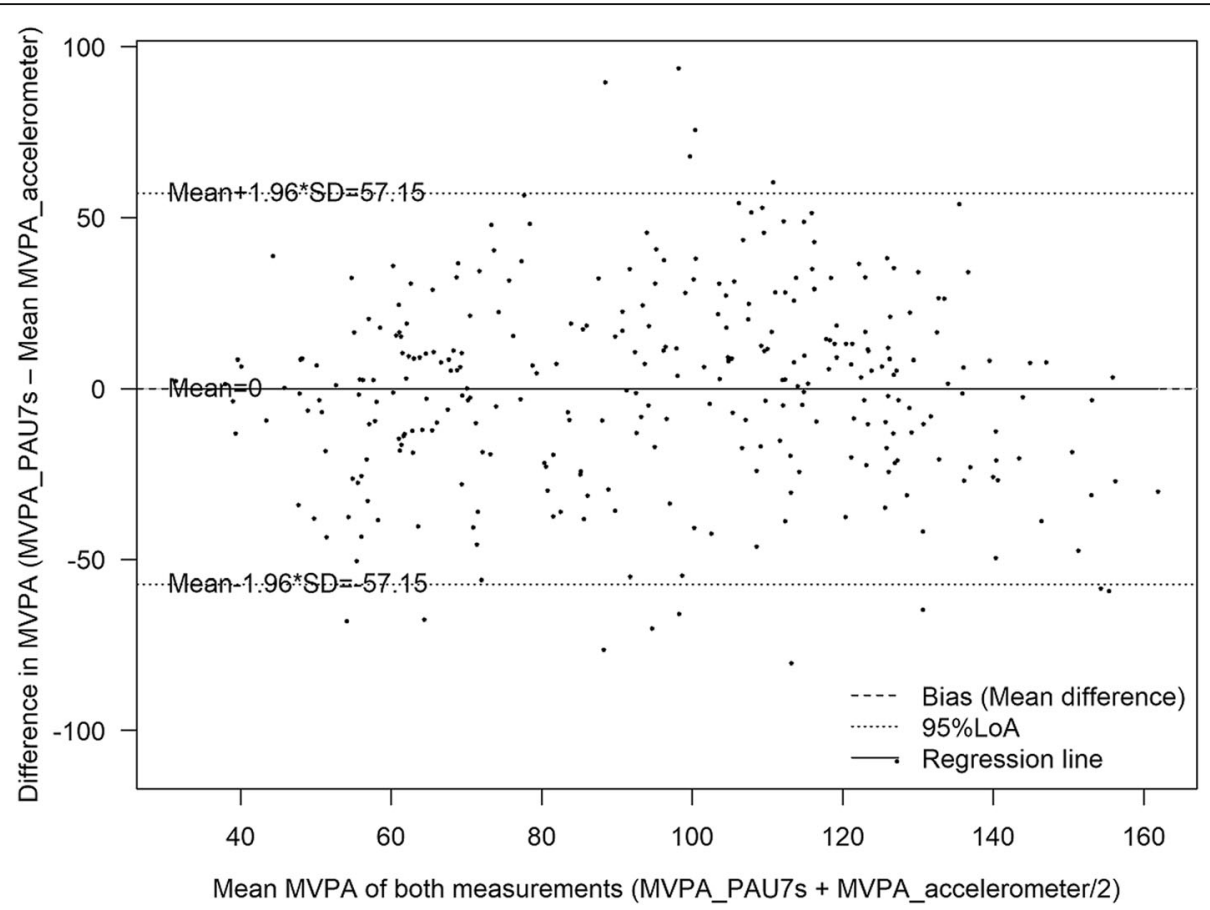

Fig. 2 Bland-Altman plot for the agreement of moderate-to-vigorous physical activity (MVPA) derived from the calibrated Physical Activity Unit 7 item Screener (PAU-7S) and the accelerometer $(N=301)$

showed a good test-retest reliability for the Spanish version of the Youth Activity Profile (YAP) questionnaire [38]. The YAP questionnaire was administered 2 weeks apart, yielding an ICC of 0.66 and 0.72 in children and adolescents, respectively. The ICC of the test-retest reliability of the PAU-7S after 9 days is comparable to that found by Martínez-Gómez and colleagues for the Spanish version of the PAQ-A (for adolescents), administered with a 1-week retest timeframe [36]. Furthermore, the PAU-7S showed considerably better test-retest

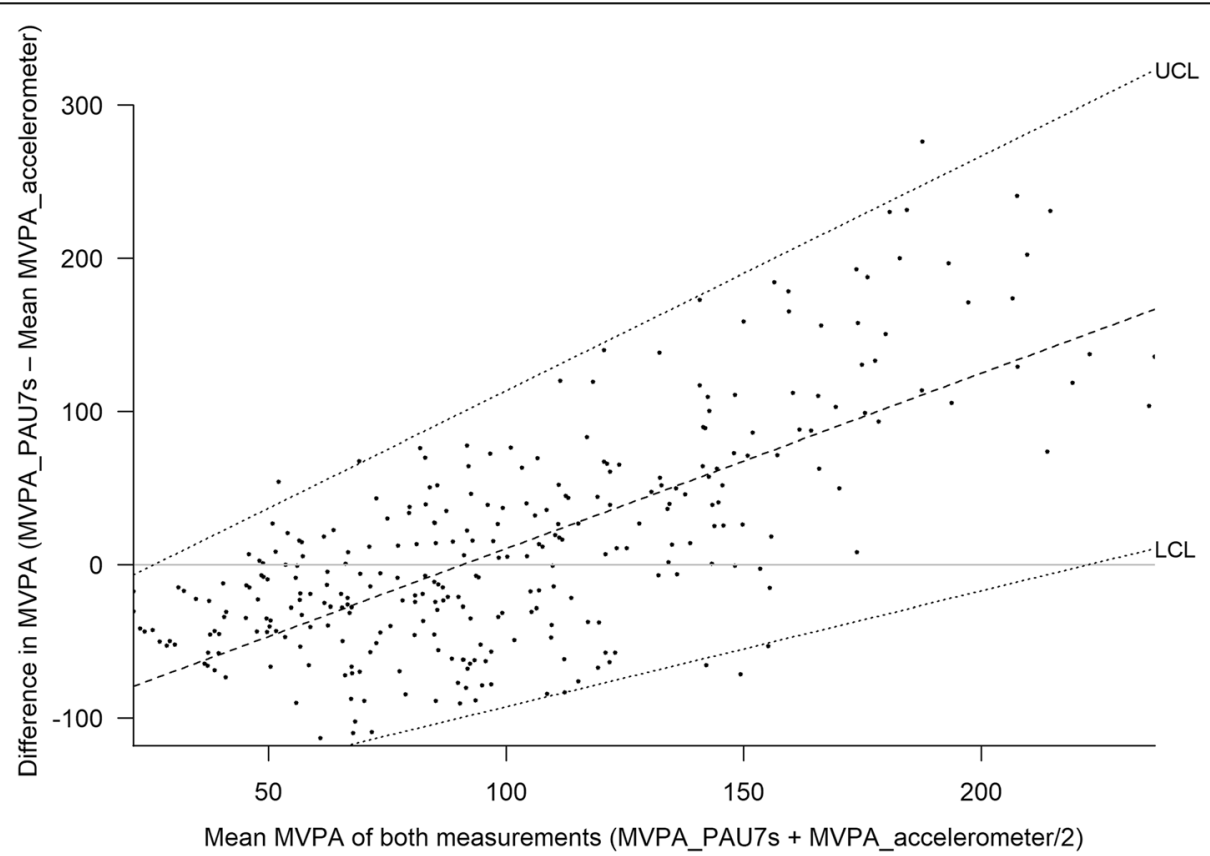

Fig. 3 Bland-Altman plot according to Ludbrook (28) for the agreement of moderate-to-vigorous physical activity (MVPA) derived from the uncalibrated Physical Activity Unit 7 item Screener (PAU-7S) and the accelerometer ( $N=301)$ 


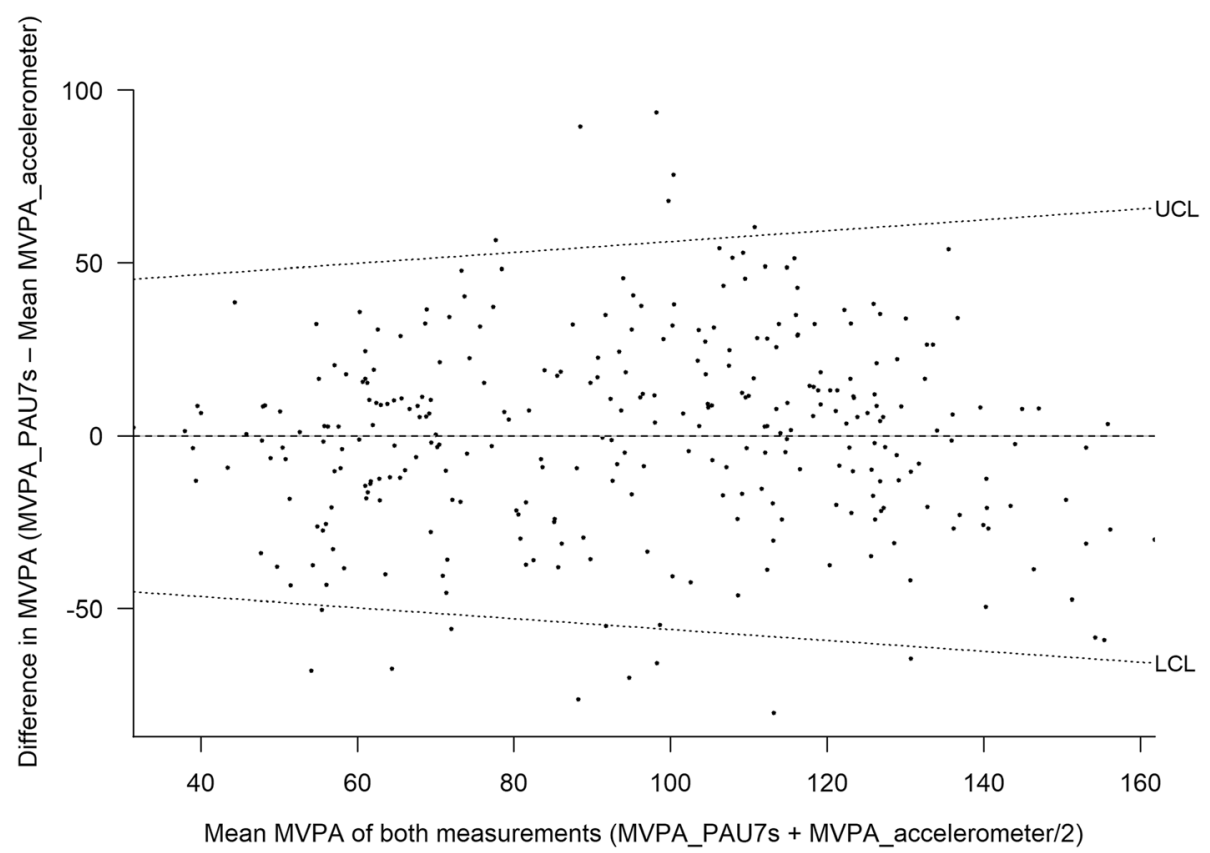

Fig. 4 Bland-Altman plot according to Ludbrook (28) for the agreement of moderate-to-vigorous physical activity (MVPA) derived from the calibrated Physical Activity Unit 7 item Screener (PAU-7S) and the accelerometer $(N=301)$

reliability compared to the short form (7 items) of the International Physical Activity Questionnaire (IPAQ; IPAQ-SF) administered in Norwegian adolescents [39].

In the noncalibrated PAU-7S data, MVPA was overestimated by $11.4 \mathrm{~min}$ per day in comparison to accelerometer-derived MVPA. This is considerably lower than the IPAQ-A overestimation by $39.8 \mathrm{~min}$ of MVPA in Spanish adolescents [40]. Furthermore, the IPAQ-SF overestimates MVPA in a range from 36 to $173 \%$ in five studies, whereas one study reports an underestimation of $28 \%$ [41].

The Spearman correlation of 0.31 for concurrent validity in the present study falls within the range reported for most PA questionnaires for youth [22,33]; we would note that few PA questionnaires for children and adolescents have been validated in the Spanish population (33, $34,37,39,40)$. However, the information yield by most of these questionnaires is limited due to the few PA domains included, as several ask only one question about out-of-school sport activities, or total PA during the day, or yield data for a qualitative comparison of PA level with that of other children (37). The concurrent validity of the PAQ-A and PAQ-C administered in Spanish adolescents and children, respectively, ranged from fair to moderate $(31,34,37)$. The highest concurrent validity $(r=0.54)$ was found for the adapted version of the Assessment of Physical Activity Levels Questionnaire (APALQ) among Spanish adolescents [10]. The PatientCentered Assessment and Counselling for Exercise Plus Nutrition (PACE+) is a two-item PA questionnaire developed to estimate compliance with the PAGuidelines for youth (1). The 60-min MVPA composite of the PACE+ showed fair to moderate validity for girls and boys, respectively, when compared to accelerometer-derived MVPA.

In most of the previously published studies, determination of the validity of the questionnaires used was limited to the assessment of concurrent validity by Spearman or Pearson correlation coefficients. This analysis can yield insights into the capacity of a questionnaire to rank children according to PA levels (e.g., low, medium, or high) but cannot assess absolute agreement between the PA questionnaire and the criterion standard of validity. In the present study, we found a somewhat lower ICC of MVPA between methods compared to that reported by Martín-Bello and colleagues [40] for the IPAQ-A. The absolute agreement of $46.7 \%$ correctly classified adolescents according to tercile classification of MVPA by our questionnaire and by accelerometerderived MVPA, in addition to a kappa value of 0.24; this indicates a fair agreement for the non-calibrated PAU7S. Furthermore, the Bland-Altman plot revealed a significant bias across the range of MVPA estimates between questionnaire and accelerometer data, showing an increasing measurement error at higher levels of MVPA on the PAU-7S.

Relatively few studies have addressed this issue for self-reported PA assessment in children (11-16). In our study, Pearson and Spearman correlation coefficients increased to 0.63 and 0.62 , respectively, after calibration of 
PAU-7S-derived MVPA estimates. Absolute agreement between methods was moderate $(\mathrm{kappa}=0.50)$ for calibrated estimates of MVPA. Furthermore, the beta coefficient of the association between MVPA derived by the PAU-7S and the WHtR and zBMI score increased from -0.010 to -2.46 and from -0.162 to -5.850 , respectively. This finding indicates a considerable improvement of the construct validity of the PAU-7S after calibration. Finally, the predictive validity of the calibrated PAU-7S data was good, according to crossvalidation with an independent internal sample. These results clearly show that linear calibration of MVPA derived by the PAU-7S strongly improved all validity dimensions tested. This finding is in line with the scarce evidence from other PA questionnaire calibration studies in children $(11,12,14-16)$. Saint-Maurice and colleagues found a reasonable ability of the PAQ$\mathrm{C}$ and PAQ-A calibration algorithm to estimate group-level estimates of accelerometer activity (15). Similar results were reported for the YAP questionnaires (14), the Global Physical Activity Questionnaire (12), and the Previous Day Physical Activity Recall (11) calibration algorithm.

The main strength of the present study is the population-based design, which permits generalization of the results to other Spanish populations of children aged 8-16 years. The inclusion of multiple dimensions of validity -concurrent, construct, and predictivealso can be considered a strength of this study. The short length of the PAU-7S makes it ideal for timelimited settings such as primary care centres and for large epidemiological studies or those that attempt to evaluate a long list of indicators. In addition, it will be useful for monitoring national PA data and for comparison with other countries. Finally, these results provide evidence that calibration can improve the validity of PA questionnaires for children and adolescents. This study also has two limitations that must be noted. Accelerometers are not sensitive to PA such as cycling or aquatics (7), and measurement error of self-reported data is an inherent limitation of questionnaires. Furthermore, the PAU-7S includes only 6 general questions about physical activities, which allows only a rough overview of the PA pattern and of METs spent in physical activities. For example, METs of sport activities are specific for each sport but the PAU-7S asks globally for time spent in team and individual sport activities. Therefore, the PAU-7S contribution to calculating the corresponding METs of these activities is limited. Hence, it should not be used when a more exact estimation of METs is the purpose of the study. Furthermore, a single administration of the PAU-7S will not accurately reveal seasonal variation in physical activities.

\section{Conclusion}

The PAU-7S is a valid instrument for the measurement of physical activity in Spanish children and adolescents aged 8 to 16 years. The questionnaire is an adequate instrument for a general estimation of PA, especially in time-limited settings such as primary care and in epidemiological surveys with a large sample size or with many measures of other health indicators where the administration of accelerometers is not feasible. The calibration of this questionnaire meaningfully decreased measurement error and thereby increased its validity. Further studies are needed to shed light on the external validity of the PAU-7S.

\section{Supplementary Information}

The online version contains supplementary material available at https://doi. org/10.1186/s12966-021-01169-w.

Additional file 1 Supplementary Table 1. Characteristics of the validation study participants and the remaining participants of the population-based PASOS cohort.

Additional file $\mathbf{2}$ Supplementary Table 2. Correlation coefficients and between-method agreement of moderate to vigorous physical activity measurements derived by the Physical Activity Unit 7-item screener, noncalibrated and calibrated, and the reference method (accelerometer), stratified by age.

\section{Acknowledgements \\ We thank the staff, pupils, parents, schools and municipalities for their participation, enthusiasm, and support. We appreciate the English revision by Elaine M Lilly, PhD.}

\section{Authors' contributions}

HS and SFG developed the questionnaire and designed the study. IS, HS and JW performed the statistical analysis. HS interpreted the data and wrote the first draft. All authors defined the strategy to deploy the PASOS study protocol. SFG, CH, and MS provided funding. All authors with the exception of HS collected data. All authors critically reviewed and edited the manuscript and provided final approval for the submitted version.

\section{Funding}

PASOS study has been funded mainly by Fundación PROBITAS and Gasol Foundation. Additional funds were received from the Barça Foundation, Banco Santander, Grupo IFA, Viena and Fundación Deporte Jóven, grant of support to research groups number 35/2011 (Balearic Islands Gov) and EU COST Action CA16112. J.A.T. and M.M.B. are funded by the official funding agency for biomedical research of the Spanish government, Institute of Health Carlos III (ISCIII), which is cofunded by the 333 European Regional Development Fund (CIBEROBN CB12/03/30038). PASOS has the institutional support of Spain's Ministry of Education and Vocational Training, the Ministry of Health, Consumption and Social Welfare through the Spanish Agency for Food Safety and Nutrition (ASEAN), the High Commission against Child Poverty, the High Sports Council, the General College of Professional Associations of Physical Education and Sports, and the Departments of Education and/or Health and/or Sports of Spain's 17 autonomous regions. The CIBERESP and the CIBEROBN are initiatives of the Institute of Health Carlos III, Madrid, Spain.

\section{Availability of data and materials}

The dataset from the current analysis is not publicly but is available from the corresponding author upon reasonable request. 


\section{Declarations}

\section{Ethics approval and consent to participate}

The study protocol was approved by the Ethics Committee CElm Fundació Sant Joan de Déu, Spain (Approval number: PIC-171-18). Parental written informed consent was obtained.

\section{Consent for publication}

Not applicable.

\section{Competing interests}

Non declared. The funders had no role in the analysis or interpretation of the data of this study.

\section{Author details}

Ciber Epidemiology and Public Health (CIBERESP), Instituto de Salud Carlos III, Madrid, Spain. ${ }^{2}$ Cardiovascular Risk and Nutrition Research Group, IMIM (Hospital del Mar Medical Research Institute), Barcelona, Spain. ${ }^{3}$ Cardiovascular Epidemiology and Genetics Research Group, IMIM Hospital del Mar Medical Research Institute, Barcelona, Spain. ${ }^{4}$ Centro de Investigación Biomédica en Red de Enfermedades Cardiovasculares (CIBERCV), Madrid, Spain. ${ }^{5}$ School of Health Sciences, Universidad de Málaga-Instituto de investigación biomédica de Málaga, Málaga, Spain. ${ }^{6}$ Centro de Investigación Biomédica en Red Fisiopatología de la Obesidad y la Nutrición (CIBEROBN), Institute of Health Carlos III, Madrid, Spain. ${ }^{7} E L I K O S$ group, Institute for Innovation \& Sustainable Development in Food Chain (IS-FOOD), Instituto de Investigación Sanitaria de Navarra (IDISNA), Public University of Navarra, Navarra, Spain. ${ }^{8}$ ImFINE Research Group, Department of Health and Human Performance, Universidad Politécnica de Madrid, Madrid, Spain. ${ }^{9}$ Physical Activity and Quality of Life Research Group (AFYCAV), Faculty of Sport Sciences, University of Extremadura, Cáceres, Spain. ${ }^{10}$ PAFS Research Group, Faculty of Sports Sciences, University of Castilla-La Mancha, Toledo, Spain. ${ }^{11} \mathrm{CIBER}$ of Frailty and Healthy Aging (CIBERFES), Madrid, Spain.

${ }^{12}$ Research Center for High Performance Sport, Catholic University of Murcia, Murcia, Spain. ${ }^{13}$ Faculty of Sport Sciences, UCAM, Catholic University of Murcia, Murcia, Spain. ${ }^{14}$ Faculty of Sports Sciences and Physical Education, University of A Coruña, A Coruña, Spain. ${ }^{15}$ Research Institute of Biomedical and Health Sciences (IUIBS), University of Las Palmas de Gran Canaria, Las Palmas, Spain. ${ }^{16}$ Preventive Medicine Service, Canarian Health Service, Centro Hospitalario Universitario Insular Materno Infantil (CHUIMI), Las Palmas, Spain. ${ }^{17}$ Regional Unit of Sports Medicine, Municipal Sports Foundation of Avilés, Asturias, Spain. ${ }^{18}$ Research Group of Community Nutrition \& Oxidative Stress, University of the Balearic Islands, Palma de Mallorca, Spain. ${ }^{19}$ Probitas Foundation, Barcelona, Spain. ${ }^{20}$ Gasol Foundation, 08830 Sant Boi de Llobregat, Spain. ${ }^{21} \mathrm{GRoW}$, Global Research on Wellbeing, Blanquerna School of Life Sciences, University Ramon Llull, Barcelona, Spain. ${ }^{22}$ Faculty of Health Sciences, Universitat Oberta de Catalunya, Barcelona, Spain. ${ }^{23}$ Department of Didactics of Language, Arts and Physical Education, Universidad Complutense de Madrid, Madrid, Spain. ${ }^{24} \mathrm{GREpS}$, Health Education Research Group, Nursing and Physiotherapy Department, University of Lleida, Lleida, Spain.

\section{Received: 15 February 2021 Accepted: 5 July 2021}

Published online: 17 July 2021

\section{References}

1. Joan Poitras V, Ellen Gray C, Borghese MM, Carson V, Chaput J-P, Janssen I, et al. Systematic review of the relationships between objectively measured physical activity and health indicators in school-aged children and youth 1. [cited 2020 Nov 25]; Available from: http://nrcresearchpress.com/doi/suppl/1 0.1139/apnm-2015-0663.

2. Hillman $\mathrm{CH}$, McDonald KM, Logan NE. A Review of the Effects of Physical Activity on Cognition and Brain Health across Children and Adolescence. Nestle Nutr Inst Workshop Ser [Internet]. Nestle Nutr Inst Workshop Ser; 2020 [cited 2020 Nov 25]. p. 1-11. Available from: https://pubmed.ncbi.nlm. nih.gov/33161407/

3. Whooten R, Kerem L, Stanley T. Physical activity in adolescents and children and relationship to metabolic health [Internet]. Curr. Opin. Endocrinol. Diabetes Obes. Lippincott Williams and Wilkins; 2019 [cited 2020 Oct 26]. p. 25-31. Available from: https://pubmed.ncbi.nlm.nih.gov/30507695/
4. Global recommendations on physical activity for health [Internet]. [cited 2020 Nov 25]. Available from: https://www.who.int/publications/i/item/ 9789241599979

5. Parrish AM, Tremblay MS, Carson S, Veldman SLC, Cliff D, Vella S, et al. Comparing and assessing physical activity guidelines for children and adolescents: A systematic literature review and analysis [Internet]. Int. J. Behav. Nutr. Phys. Act. BioMed Central; 2020 [cited 2020 Oct 26]. p. 16. Available from: https://ijbnpa.biomedcentral.com/articles/10.1186/s12966-02 0-0914-2

6. Lobelo F, Rohm Young D, Sallis R, Garber MD, Billinger SA, Duperly J, et al. Routine Assessment and Promotion of Physical Activity in Healthcare Settings: A Scientific Statement From the American Heart Association. Circulation [Internet]. NLM (Medline); 2018 [cited 2020 Oct 26];137:e495-522. Available from: http://circ.ahajournals.org

7. Warren JM, Ekelund U, Besson H, Mezzani A, Geladas N, Vanhees L. Assessment of physical activity - A review of methodologies with reference to epidemiological research: A report of the exercise physiology section of the European Association of Cardiovascular Prevention and Rehabilitation [Internet]. Eur. J. Cardiovasc. Prev. Rehabil. SAGE Publications Inc.; 2010 [cited 2020 Oct 26]. p. 127-39. Available from: https://pubmed.ncbi.nIm.nih. gov/20215971/

8. Migueles JH, Cadenas-Sanchez C, Tudor-Locke C, Löf M, Esteban-Cornejo I, Molina-Garcia P, et al. Comparability of published cut-points for the assessment of physical activity: implications for data harmonization. Scand J Med Sci Sports. 2019;29:566-74.

9. Prochaska JJ, Sallis JF, Long B. A Physical Activity Screening Measure for Use With Adolescents in Primary Care. Arch Pediatr Adolesc Med. 2001.

10. Zaragoza Casterad J, Generelo E, Aznar S, Abarca-Sos A, Julián JA, Mota J. Validation of a short physical activity recall questionnaire completed by Spanish adolescents. Eur J Sport Sci. 2012;12(3):283-91. https://doi.org/10.1 080/17461391.2011.566357.

11. Saint-Maurice PF, Welk GJ. Validity and calibration of the youth activity profile. PLoS One. 2015;10:1-16.

12. Rääsk T, Maëstu J, Lätt $E$, Jürimäe J, Jürimäe T, Vainik U, et al. Comparison of IPAQ-SF and two other physical activity questionnaires with accelerometer in adolescent boys. PLoS One. 2017;12:1-14.

13. Pate RR, Hillman CH, Janz KF, Katzmarzyk PT, Powell KE, Torres A, et al. Physical Activity and Health in Children Younger than 6 Years: A Systematic Review [Internet]. Med. Sci. Sports Exerc. Lippincott Williams and Wilkins; 2019 [cited 2020 Oct 26]. p. 1282-91. Available from: https://pubmed.ncbi. nlm.nih.gov/31095085/

14. Tucker JM, Welk G, Nusser SM, Beyler NK, Dzewaltowski D. Estimating minutes of physical activity from the previous day physical activity recall: validation of a prediction equation. J Phys Act Health. 2011;8(1):71-8. https://doi.org/10.1123/jpah.8.1.71.

15. Metcalf KM, Baquero BI, Coronado Garcia ML, Francis SL, Janz KF, Laroche $\mathrm{HH}$, et al. Calibration of the global physical activity questionnaire to Accelerometry measured physical activity and sedentary behavior. BMC Public Health BMC Public Health. 2018;18:1-10.

16. Saint-Maurice PF, Kim Y, Hibbing P, Oh AY, Perna FM, Welk GJ. Calibration and Validation of the Youth Activity Profile: The FLASHE Study. Am J Prev Med [Internet]. Elsevier Inc.; 2017;52:880-7. Available from: https://doi.org/1 0.1016/jamepre.2016.12.010

17. Saint-Maurice PF, Welk GJ, Beyler NK, Bartee RT, Heelan KA. Calibration of self-report tools for physical activity research: the physica............................................. activity questionnaire (PAQ). BMC Public Health. 2014;14:1-9.

18. Fairclough SJ, Christian DL, Saint-Maurice PF, Hibbing PR, Noonan RJ, Welk $\mathrm{GJ}$, et al. Calibration and validation of the youth activity profile as a physical activity and sedentary behaviour surveillance tool for english youth. Int J Environ Res Public Health. 2019;16(19). https://doi.org/10.3390/ijerph16193711.

19. Gómez SF, Homs C, Wärnberg J, Medrano M, Gonzalez-Gross M, Gusi N et al. Study protocol of a population-based cohort investigating physical activity, Sedentarism, lifestyles and obesity in Spanish youth: the PASOS study. BMJ Open. 2020;10:1-6.

20. Gomez SF, Casas R, Palomo VT, Pujol AM, Fíto M, Schröder H. Study protocol: effects of the THAO-child health intervention program on the prevention of childhood obesity - the POIBC study [internet]. 2014. Available from: http://www.biomedcentral.com/1471-2431/14/215

21. Serra Majem L, Ribas Barba L, Aranceta Bartrina J, Pérez Rodrigo C, Saavedra Santana P, Peña Quintana L. Childhood and adolescent obesity in Spain. 
Results of the enKid study (1998-2000). Med Clin (Barc) [Internet]. Ediciones Doyma, S.L.; 2003 [cited 2020 Nov 25];121:725-32. Available from: https:// pubmed.ncbinlm.nih.gov/14678693/

22. Chinapaw MJM, Mokkink LB, Van Poppel MNM, Van Mechelen W, Terwee CB. Physical activity questionnaires for youth: a systematic review of measurement properties. Sports Med. 2010;40(7):539-63. https://doi.org/1 0.2165/11530770-000000000-00000

23. Troiano RP, Berrigan D, Dodd KW, Mâsse LC, Tilert T, Mcdowell M. Physical activity in the United States measured by accelerometer. Med Sci Sports Exerc [Internet]. Med Sci Sports Exerc; 2008 [cited 2020 Nov 25];40:181-8. Available from: https://pubmed.ncbi.nIm.nih.gov/18091006/

24. Chandler JL, Brazendale K, Beets MW, Mealing BA. Classification of physical activity intensities using a wrist-worn accelerometer in 8-12-year-old children. Pediatr Obes. 2016;11(2):120-7. https://doi.org/10.1111/ijpo.12033.

25. De Onis M, Onyango AW, Borghi E, Siyam A, Nishida C, Siekmann J. Development of a WHO growth reference for school-aged children and adolescents. Bull World Health Organ. 2007;85(09):660-7. https://doi.org/1 0.2471/BLT.07.043497.

26. Akoglu H. User's guide to correlation coefficients [Internet]. Turkish J. Emerg. Med. Emergency Medicine Association of Turkey; 2018 [cited 2020 Nov 12]. p. 91-3. Available from: /pmc/articles/PMC6107969/?report=abstract.

27. Altman DG, Bland JM. Measurement in medicine: the analysis of method comparison studies. Stat. 1983;32:307.

28. Ludbrook J. Confidence in Altman-Bland plots: A critical review of the method of differences [Internet]. Clin. Exp. Pharmacol. Physiol. Clin Exp Pharmacol Physiol; 2010 [cited 2021 Jun 25]. p. 143-9. Available from: https://pubmed.ncbi.nlm.nih.gov/19719745/

29. Corder K, Van Sluijs EMF, Wright A, Whincup P, Wareham NJ, Ekelund U. Is it possible to assess free-living physical activity and energy expenditure in young people by self-report? Am J Clin Nutr. 2009;89(3):862-70. https://doi. org/10.3945/ajcn.2008.26739.

30. Shook RP. Obesity and energy balance: What is the role of physical activity? [Internet]. Expert Rev. Endocrinol. Metab. Taylor and Francis Ltd; 2016 [cited 2020 Nov 13]. p. 511-20. Available from: https://pubmed.ncbi.nlm.nih.gov/3 0058919/

31. Rosner B, Willett WC, Spiegelman D. Correction of logistic regression relative risk estimates and confidence intervals for systematic within-person measurement error. Stat Med [Internet]. Stat Med; 1989 [cited 2020 Nov 4];8: 1051-69. Available from: https://pubmed.ncbi.nlm.nih.gov/2799131/

32. Hidding LM, Chinapaw MJM, van Poppel MNM, Mokkink LB, Altenburg TM. An Updated Systematic Review of Childhood Physical Activity Questionnaires [Internet]. Sport. Med. Springer International Publishing; 2018. Available from: https://doi.org/10.1007/s40279-018-0987-0, 48, 12, $2797,2842$.

33. Helmerhorst HJF, Brage S, Warren J, Besson H, Ekelund U. A systematic review of reliability and objective criterion-related validity of physical activity questionnaires. Int J Behav Nutr Phys Act. 2012;9(1):103. https://doi.org/10.11 86/1479-5868-9-103.

34. Price RJ, Chiang I-CA. Reliability and Validity of Measurement Scales. Physiotherapy Theory and Practice, 2012, 28(3), 188-197.

35. Using and Interpreting Cronbach's Alpha | University of Virginia Library Research Data Services + Sciences [Internet]. [cited 2020 Nov 26]. Available from: https://data.library.virginia.edu/using-and-interpreting-cronbachs-a Ipha/

36. Martínez-Gómez D, Martínez-De-Haro V, Del-Campo J, Zapatera B, Welk GJ, Villagra A, et al. Validez de cuatro cuestionarios para valorar la actividad física en adolescentes españoles. Gac Sanit. 2009;23:512-7.

37. Benítez-Porres J, López-Fernández I, Raya JF, Álvarez Carnero S, Alvero-Cruz $\mathrm{JR}$, Álvarez CE. Reliability and validity of the PAQ-C questionnaire to assess physical activity in children. J Sch Health. 2016;86(9):677-85. https://doi. org/10.1111/josh.12418.

38. Segura-Díaz JM, Barranco-Ruiz Y, Saucedo-Araujo RG, Aranda-Balboa MJ, Cadenas-Sanchez C, Migueles JH, et al. Feasibility and reliability of the Spanish version of the youth activity profile questionnaire (YAP-Spain) in children and adolescents. J sports Sci [internet]. Routledge; 2020;00:1-7. Available from: https://doi.org/10.1080/02640414.2020.1847488, 2021.

39. Rangul V, Holmen TL, Kurtze N, Cuypers K, Midthjell K. Reliability and validity of two frequently used self-administered physical activity questionnaires in adolescents. BMC Med Res Methodol. 2008;8:1-10.

40. Mart C, Casaj JA. Validación de los cuestionarios PAQ-C e IPAQ-A en niños/ as en edad escolar. Cult Cienc y Deport. 2020;15:177-87.
41. Lee PH, Macfarlane DJ, Lam T, Stewart SM. Validity of the international physical activity questionnaire short form. Int J Behav Nutr Phys Act. 2011;8: $1-11$.

\section{Publisher's Note}

Springer Nature remains neutral with regard to jurisdictional claims in published maps and institutional affiliations.
Ready to submit your research? Choose BMC and benefit from:

- fast, convenient online submission

- thorough peer review by experienced researchers in your field

- rapid publication on acceptance

- support for research data, including large and complex data types

- gold Open Access which fosters wider collaboration and increased citations

- maximum visibility for your research: over $100 \mathrm{M}$ website views per year

At BMC, research is always in progress.

Learn more biomedcentral.com/submissions 